\title{
Educativa
}

Núm. 20/ Enero 06

\section{La educación continúa en México: hacia la transición a la captación a distancia}

\author{
Rosario Vega García \\ Universidad Nacional Autónoma de México. \\ vegarosario@yahoo.com
}

Resumen: Los grandes cambios que se han suscitado en la educación a distancia en los últimos tiempos, han sido impulsados por distintas variables culturales, sociales, políticas y económicas. En México también se han hecho presentes estas transformaciones, apoyadas por los esfuerzos de las autoridades educativas, investigadores del sector y docentes para buscar y aplicar modalidades educativas que permitan una mayor cobertura.

Abstract: The big changes that have been provoked in the education distantly in the last times, have been stimulated by different cultural, social, political and economic variables. In Mexico also there have become present these transformations supported by the efforts, investigative of the educational authorities of the sector and teachers to seek and to apply educational modalities that allow a major coverage.

Palabras claves: Educación Continua / Educación a Distancia/ Tecnologías de la Información y la Comunicación.

Key Words: Education Continues / Education to Distancia/ Tecnologías of the Information and the Communication.

\section{INTRODUCCIÓN.}

¿Por qué la educación a distancia en México?

Entre las modalidades dentro del ámbito de la educación superior, la educación a distancia en México se está ofertando en diferentes instituciones como una opción viable, para sortear 
algunas de las barreras que la formación presencial no puede superar, como son: la cuestión geográfica, la imposibilidad de cubrir el tiempo preestablecido de permanencia en un centro educativo, y cuestiones relacionadas con los aspectos sociales, culturales, económicos y profesionales del estudiante.

"Los jóvenes que provienen de grupos en situación de marginación se enfrentan a serios obstáculos para tener acceso a la educación superior, permanecer en ella y graduarse oportunamente. Mientras que el 45\% del grupo de edad entre 19 y 23 años, que vive en zonas urbanas y pertenece a familias con ingresos medios o altos recibe educación superior, únicamente el 11\% de quienes habitan en sectores urbanos pobres y el 3\% de los que viven en sectores rurales pobres cursan este tipo de estudios. Por su parte, la participación de los estudiantes indígenas es mínima." [1]

Entre las conclusiones a las que llegaron los participantes del seminario Universidades Virtuales en América Latina y Caribe, organizado por la UNESCO, UNIVERSIA y el Gobierno de Ecuador, cabe destacar la necesidad de ampliar el acceso a una educación superior de calidad en Latinoamérica, con la finalidad de contribuir al bienestar socioeconómico.[2]

Ante este panorama no es de extrañar que en el Programa Nacional de Educación 2001 - 2006 se contemple como meta a alcanzar, el incremento de la matrícula en programas de educación superior abierta, semi presencial y a distancia de 149.809 en el 2000 a 200.000 en el 2006.[3]

\section{EL PAPEL DE LAS TECNOLOGÍAS DE LA INFORMACióN Y LA COMUNICACIÓN EN LA EDUCACIÓN SUPERIOR A DISTANCIA.}

Uno de los principales factores que ha impulsado la rápida evolución de esta modalidad en la educación superior, ha sido la incursión de las tecnologías de la información y la comunicación en la educación. Esto ha permitido la incorporación del uso del vídeo y de la televisión, que junto con los sistemas satelitales y la fibra óptica, posibilitan la transmisión de las telesesiones y las videoconferencias dentro del ámbito educativo. El impacto del desarrollo de la informática ha tenido sus implicaciones en la educación a través del uso de la internet y del CD-ROM, dándose la tendencia actual a integrar diferentes tecnologías como lo demuestran la generación de portales WAP, que permiten el acceso a frecuencias de radio y de televisión vía internet a través de la telefonía móvil y la creación de portales PDA, que posibilitan el acceso a cursos virtuales, videoconferencias, así como consultar bases de datos, y visitar otro tipo de actos académicos.

Estas herramientas con posibilidades sincrónicas y asincrónicas, propician nuevas opciones de interacción y retroalimentación, cuyas implicaciones se traducen en modificaciones en torno al tiempo y el espacio de la participación de los profesores y los estudiantes en el proceso enseñanza - aprendizaje, ofreciendo múltiples posibilidades de aplicación en la educación, siempre y cuando no se pierda de vista que se trata de un 
medio y no de un fin en sí mismo.

El uso didáctico de estas tecnologías implica una reorganización en el tratamiento de los contenidos, requiriendo la elaboración del diseño instruccional y de guías didácticas para la obtención del cumplimiento de los objetivos formativos de cada programa, lo que supone un cambio de paradigma para todos los actores involucrados. Concretamente el profesor pasa de ser facilitador del aprendizaje a mediador del proceso de enseñanza aprendizaje, en un proceso en el que se transforma la información en conocimiento, presentando un nuevo panorama de enseñanza - aprendizaje.

\begin{tabular}{|c|c|c|c|c|}
\hline & $\begin{array}{l}\text { Experiencia previa } \\
\text { (estudiante) }\end{array}$ & $\begin{array}{l}\text { Papel del } \\
\text { estudiante }\end{array}$ & \begin{tabular}{|l|} 
Orientación del \\
estudio
\end{tabular} & Motivación \\
\hline Profesor & Hábitos de estudio. & $\begin{array}{ll}\text { Seguir } & \text { las } \\
\text { indicaciones } & \text { del } \\
\text { profesor } & \\
\text { expuestas } & \text { en } \\
\text { clase. } & \end{array}$ & $\begin{array}{|ll|}\text { Seguir } & \text { las } \\
\text { indicaciones } & \text { del } \\
\text { profesor } & \\
\text { expuestas } & \text { en } \\
\text { clase. } & \\
\end{array}$ & $\begin{array}{l}\text { Interacción y } \\
\text { retroalimentación } \\
\text { con el alumno en el } \\
\text { aula. }\end{array}$ \\
\hline Asesor & $\begin{array}{l}\text { Conocimientos en el } \\
\text { uso de las } \\
\text { tecnologías de la } \\
\text { información y la } \\
\text { comunicación. } \\
\text { Hábitos de estudio. }\end{array}$ & $\begin{array}{l}\text { Estudio } \\
\text { independiente. } \\
\text { Autodisciplina. }\end{array}$ & $\begin{array}{l}\text { Seguir las } \\
\text { indicaciones } \\
\text { expuestas en la guía } \\
\text { de estudio. }\end{array}$ & 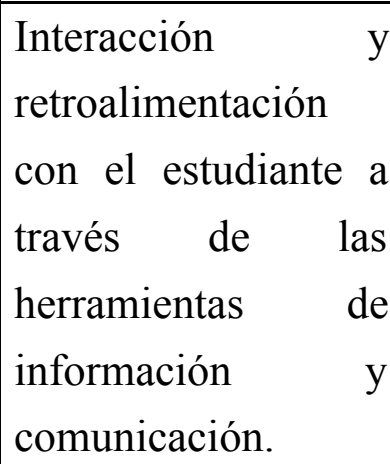 \\
\hline
\end{tabular}

\begin{tabular}{|l|l|l|l|}
\cline { 2 - 5 } \multicolumn{1}{c|}{} & Ambiente & Horario & Evaluación \\
\hline \multirow{2}{*}{ Profesor } & $\begin{array}{l}\text { Ambiente de enseñanza }- \\
\text { aprendizaje en el aula. }\end{array}$ & Inflexible & $\begin{array}{l}\text { A través de observación, } \\
\text { pruebas presenciales, resolución } \\
\text { de problemas, evaluación } \\
\text { permanente... }\end{array}$ \\
\hline & $\begin{array}{l}\text { Ambiente de enseñanza }- \\
\text { aprendizaje apoyado en } \\
\text { los multimedios. }\end{array}$ & Flexible & $\begin{array}{l}\text { A través de resolución de } \\
\text { problemas, evaluación } \\
\text { permanente, evaluación } \\
\text { automatizada... }\end{array}$ \\
\hline
\end{tabular}

A su vez, el uso de estas herramientas ofrece un planteamiento nuevo en cuanto a la organización educativa, donde podrá incluirse instrumentos de gestión escolar automatizados, que permitan una administración flexible, con una rápida respuesta a las demandas de capacitación. 
comunicación en la educación se han ido formando dos puntos de opinión enfrentados, protagonizados por un lado por los defensores más entusiastas, que perciben la aplicación de estas herramientas como una respuesta a las necesidades sociales, que a su vez están gestando nuevos paradigmas culturales y, por otro lado, se encuentra el grupo de quienes centran su atención en los efectos que sufrirán los sectores sociales excluidos del uso de estas tecnologías.[4]

\section{PANORAMA DE LA EDUCACIÓN CONTINUA EN MÉXICO.}

Tras la Segunda Guerra Mundial se produce una acelerada transformación de la economía occidental, que demandaría una mano de obra cada vez más especializada dentro de los diferentes sectores, y por tanto, de una formación adecuada a los diferentes requerimientos exigidos por el mercado laboral. Ante estas necesidades se solicitaba una oferta educativa variada, que en primera instancia las universidades no cubrían. Como producto de estos acontecimientos surgen, al término de la última gran guerra, organizaciones internacionales dedicadas a la atención de la educación y la cultura.

Más significativo es cómo, a raíz del fuerte crecimiento industrial producido a partir de los años sesenta, proliferan las actividades de educación continua, y el hecho irrefutable es que desde aquella década y hasta la fecha, la educación dentro del mundo industrializado es considerada como permanente o para toda la vida. Las fuertes y aceleradas transformaciones en el sector tecnológico, industrial y laboral, han generado una necesidad de actualización y especialización constante que solo una adecuada oferta en capacitación puede satisfacer.

México no es la excepción, su primer antecedente se dio en las I Jornadas Médicas, celebradas en 1933 en la Universidad Nacional Autónoma de México, y no es hasta 1959 cuando se da otra actividad de esta índole en citada institución educativa, sin dejar de llamar la atención la fuerte proliferación de proyectos de educación continua en los años sesenta, setenta y ochenta.[5]

Sin duda, la educación continua forma parte del proceso de cambio de las instituciones de educación superior. La visión de la universidad como generadora y transformadora de conocimiento y cultura, se enfrenta o se complementa, según se vea, a la universidad generadora de procesos de modernización y desarrollo tecnológico que requiere actualmente el sector laboral y social. Esta última forma de interpretar y entender la educación superior es la que se ha transmitido en los planes y programas nacionales de educación desde el período presidencial de Salinas, seguido por la presidencia de Zedillo y que continúa en el período presidencial de Fox.

Los nuevos escenarios de la educación superior, entendida ésta como instrumento esencial de formación de recursos humanos, requieren de una transformación en la organización y planeación de los mismos. Ante este panorama la educación continua aportaría las bases para favorecer la vinculación entre los centros de educación superior, 
la sociedad y el sector productivo, dotando una educación permanente fuera de la educación formal, que permitiría desarrollar el potencial personal y profesional de los miembros de la sociedad.

La creación de las redes y asociaciones de educación continua son muy recientes, aunque en México, como anteriormente mencioné, las actividades de este tipo de educación se vienen dando desde los años treinta del siglo XX, lo cierto es que aparecen con mayor fuerza a partir de la generación de los años ochenta. La creación de asociaciones y redes vinculadas con la educación permanente comienza a surgir con mayor proliferación en los años noventa. En 1990 se conforma la Asociación Mexicana de Educación Continua, los programas de educación continua promovidos por COLUMBUS apenas se dan a partir de 1994 y se funda la Red Universitaria de Educación Continua de América Latina y el Caribe en 1998. Con estos antecedentes, podemos concluir que en la actualidad es cuando se están dando a conocer los primeros beneficios de estas redes y asociaciones.

Las instituciones mexicanas dedicadas a la educación continua que han incorporando la modalidad a distancia dentro de sus actividades formativas, lo han hecho ante la idea de que esta modalidad facilita la posibilidad de ampliar su oferta de capacitación.

\section{SELECCIÓN DE LAS TECNOLOGÍAS DE LA INFORMACIÓN Y LA COMUNICACIÓN EN LA CAPACITACIÓN.}

La selección de estas tecnologías deberá centrarse, entre otros aspectos a consideración, en el valor de sus aportaciones pedagógicas, en cuanto a su capacidad para cubrir necesidades y mejorar situaciones de enseñanza - aprendizaje, contribuir en el cumplimiento de los objetivos de aprendizaje, así como en el análisis de los resultados producto de los estudios de las ventajas y desventajas a la hora de aplicar cada una de estas tecnologías en la educación continua. Para ello se expone el siguiente cuadro, que servirá como guía para la reflexión en la toma de decisiones.

\section{Criterios de selección de las tecnologías de la información y la comunicación en la capacitación[6]}

\begin{tabular}{|c|c|c|}
\hline CRITERIOS & $\begin{array}{c}\text { ELEMENTOS DE } \\
\text { ANÁLISIS }\end{array}$ & INDICADORES \\
\hline Calidad & $\begin{array}{c}\text { ¿Cubre las necesidades } \\
\text { formativas? }\end{array}$ & Estándares de calidad \\
\hline Cobertura & ¿Qué tanto abarca? & Relación oferta - demanda. \\
\hline Pertinencia & $\begin{array}{c}\text { ¿Cuáles son las ventajas y los } \\
\text { inconvenientes? }\end{array}$ & Relación propuesta - realidad. \\
\hline Equidad & $\begin{array}{c}\text { ¿Qué tan desigual puede resultar } \\
\text { su aplicación? }\end{array}$ & Relación justo - injusto. \\
\hline Eficacia & ¿Alcanzaremos los objetivos & Relación costo - beneficio.
\end{tabular}




\begin{tabular}{|c|c|c|}
\hline & académicos propuestos? & \\
\hline Satisfacción & $\begin{array}{l}\text { ¿Cubre los objetivos de } \\
\text { aprendizaje propuestos? }\end{array}$ & $\begin{array}{c}\text { Relación necesidades - } \\
\text { satisfacción de necesidades. }\end{array}$ \\
\hline Impacto & $\begin{array}{l}\text { ¿Qué tan relevante es su } \\
\text { aplicación? }\end{array}$ & $\begin{array}{l}\text { Relación resultados presentes } \\
\text { - resultados futuros. }\end{array}$ \\
\hline Capacitación & $\begin{array}{c}\text { ¿Cuáles son las características del } \\
\text { instructor? }\end{array}$ & Actualización permanente. \\
\hline Perfil de usuario & $\begin{array}{c}\text { ¿Cuales son las características de } \\
\text { los estudiantes? }\end{array}$ & Formación previa. \\
\hline Recursos Humanos & $\begin{array}{c}\text { ¿Se cuenta con los recursos } \\
\text { humanos necesarios para la } \\
\text { aplicación de la nueva } \\
\text { herramienta? }\end{array}$ & $\begin{array}{l}\text { Análisis de los recursos } \\
\text { humanos. }\end{array}$ \\
\hline Recursos Técnicos & $\begin{array}{c}\text { ¿Se cuenta con los recursos } \\
\text { técnicos necesarios para la } \\
\text { aplicación de la nueva } \\
\text { herramienta? }\end{array}$ & $\begin{array}{l}\text { Análisis de los recursos } \\
\text { técnicos. }\end{array}$ \\
\hline Recursos Materiales & $\begin{array}{c}\text { ¿Se cuenta con los recursos } \\
\text { materiales necesarios para la } \\
\text { aplicación de la nueva } \\
\text { herramienta? }\end{array}$ & $\begin{array}{c}\text { Análisis de los recursos } \\
\text { materiales. }\end{array}$ \\
\hline Recursos financieros & $\begin{array}{c}\text { ¿Se cuenta con los recursos } \\
\text { financieros necesarios para la } \\
\text { adquisición de la nueva } \\
\text { herramienta? }\end{array}$ & $\begin{array}{l}\text { Análisis de los recursos } \\
\text { financieros. }\end{array}$ \\
\hline Poder & $\begin{array}{c}\text { ¿Cuál es la actitud de las } \\
\text { autoridades? }\end{array}$ & $\begin{array}{l}\text { Análisis de la actitud de } \\
\text { autoridades. }\end{array}$ \\
\hline Burocracia & $\begin{array}{c}\text { ¿Cuál es la eficacia de la } \\
\text { burocracia? }\end{array}$ & Tiempo \\
\hline
\end{tabular}

\section{LOS DESAFÍOS DE LA EDUCACIÓN CONTINUA A DISTANCIA EN MÉXICO.}

Con el uso de las tecnologías de la información y la comunicación en la educación, nos encontramos con un nuevo panorama que nos permite resolver necesidades educativas y que, a su vez, genera nuevos retos ha superar, entre los que destacan:

1.- Creación de una auténtica política educativa nacional, donde se contemple la educación superior a distancia como proyecto de nación, que facilite los mecanismos para dar respuesta a los vertiginosos cambios sociales y la toma de decisiones sobre cualquier innovación o cambio educativo. 
2.- Cambio y fortalecimiento en la estructura organizativa de los centros de educación continua. Esta circunstancia implica cambios en la gestión de los centros, comenzando por la reforma y fortalecimiento de la legislación y normatividad que los rigen, así como de la administración educativa.

3.- Conformación de una educación continua por competencias, para permitir una eficiente respuesta a las necesidades de formación.

4.- Aumento de presupuestos económicos para la obtención de recursos humanos, materiales y técnicos, y para la permanente capacitación del personal.

5.- Elaboración de estándares de calidad para el uso de las tecnologías de la información y la comunicación en la educación, con la finalidad de desarrollar prácticas reguladoras.

6.- Diseño de proyectos que garanticen el incremento de los niveles de conectividad de las instituciones, a través del desarrollo de infraestructura técnica.

7.- Creación de mecanismos adecuados de gestión de la información y el conocimiento.

8.- Dentro de esta gestión del conocimiento, es importante promover la creación e integración de grupos de trabajo de investigación, para conformar redes regionales que faciliten el tránsito de información y conocimiento.

9.- Aportar estrategias que involucren a los instructores en la aplicación de las tecnologías de la información y la comunicación en sus dinámicas formativas, de tal forma que las instituciones y los capacitadores compartan los resultados académicos.

10.- Desarrollo de programas de formación de instructores sobre el uso de las tecnologías de la información y la comunicación.

11.- Vincular la investigación con la educación continua.

12.- Propiciar la edición de publicaciones, difundir experiencias y productos de investigación.

13.- Promover una red regional de bibliotecas digitales.

14.- Generar una cultura de la capacitación en torno al uso de las tecnologías de la información y la comunicación.

\section{BIBLIOGRAFÍA}


EDUCACIÓN SUPERIOR. (2004). Estudio sobre el uso de las tecnologías de comunicación e información para la virtualización de la educación superior en México. ANUIES, México, D. F. (material mecanografiado).

ASOCIACIÓN NACIONAL DE UNIVERSIDADES E INSTITUCIONES DE EDUCACIÓN SUPERIOR. (2001). Plan maestro de educación superior abierta y a distancia. Líneas estratégicas para su desarrollo, ANUIES, México, D. F.

Declaración de Quito sobre el rol de las universidades en la sociedad de la información, UNESCO, UNIVERSIA, 13 y 14 de febrero de 2003, (documento impreso).

FERNÁNDEZ HERNÁNDEZ, P. (2002). Competencias y habilidades para el uso de nuevas tecnologías en la educación, en Foro de planeación académica, Área de Ciencias Sociales: Diálogo con expertos, Preparatoria $N^{\circ}$ 7, Monterrey, Nuevo León, 2 de agosto de 2002, (material mecanografiado).

RODRÍGUEZ, M.; EUSTAQUIO y AHIJADO QUINTILLÁN, M. (coordinadores) (1999). La educación a distancia en tiempo de cambios: nuevas generaciones viejos conflictos. Ediciones de la Torre, Madrid.

PIÑETA RAMÍREZ, D. (coord.) (2002). La Educación Superior en el Proceso Histórico de México: Tomo III. Cuestiones Esenciales. Prospectivas del Siglo XXI, Secretaría de Educación Pública, Universidad Autónoma de Baja California, Asociación Nacional de Universidades e Instituciones de Educación Superior, Baja California.

SEP, Programa Nacional de Educación 2001 - 2006, SEP, México, D. F., 2000.

VEGA GARCÍA, R. (2004). Aplicación y evaluación de las tecnologías de la información y la comunicación en la educación, en Evaluación y Tendencias en la Educación Profesional Técnica en Hispanoamérica en el siglo XXI, Colegio Nacional de Educación Profesional Técnica y Agencia Española de Cooperación Internacional, México, D. F., junio 2004. (CD-ROM).

VEGA GARCÍA, R. (2005). La educación continua a distancia en México: la Universidad Nacional Autónoma de México, en Revista de Educación Superior, Asociación Nacional de Universidades e Instituciones de Educación Superior (ANUIES), volumen XXXIV (1), enero - marzo de 2005, México, D. F.

[1]Programa Nacional de Educación 2001 - 2006, SEP, México, D. F., 2000, pp. 189.

[2] Declaración de Quito sobre el rol de las universidades en la sociedad de la información, UNESCO, UNIVERSIA, 13 y 14 de febrero de 2003, (documento impreso), pp. 1.

[3]SEP, Programa Nacional de Educación 2001 - 2006, SEP, México, D. F., 2000, pp. 203.

[4]Fernández Hernández, Patricia, "Competencias y habilidades para el uso de nuevas tecnologías en la educación”, en Foro de planeación académica, Área de Ciencias Sociales: Diálogo con expertos, Preparatoria $\mathrm{N}^{\circ} 7$, Monterrey, Nuevo León, 2 de agosto de 2002, (material mecanografiado).

[5]García González, Eva Laura y otros, Gestión de la educación continua y la capacitación, Manual Moderno, 2004, pp. $11-13$.

[6]Vega García, Rosario: “Aplicación y evaluación de las tecnologías de la información y la comunicación en la educación”, en Evaluación y Tendencias en la Educación Profesional Técnica en Hispanoamérica en el siglo XXI, Colegio Nacional de Educación Profesional Técnica y Agencia Española de Cooperación Internacional, México, D. F., junio 2004. (CD-ROM). 
o:p>

VEGA GARCÍA, R. (2005). La educación continua a distancia en México: la Universidad Nacional Autónoma de México, en Revista de Educación Superior, Asociación Nacional de Universidades e Instituciones de Educación Superior (ANUIES), volumen XXXIV (1), enero - marzo de 2005, México, D. F.

[1]Programa Nacional de Educación 2001 - 2006, SEP, México, D. F., 2000, pp. 189.

[2] Declaración de Quito sobre el rol de las universidades en la sociedad de la información, UNESCO, UNIVERSIA, 13 y 14 de febrero de 2003, (documento impreso), pp. 1.

[3]SEP, Programa Nacional de Educación 2001 - 2006, SEP, México, D. F., 2000, pp. 203.

[4]Fernández Hernández, Patricia, "Competencias y habilidades para el uso de nuevas tecnologías en la educación”, en Foro de planeación académica, Área de Ciencias Sociales: Diálogo con expertos, Preparatoria $\mathrm{N}^{\circ} 7$, Monterrey, Nuevo León, 2 de agosto de 2002, (material mecanografiado).

[5]García González, Eva Laura y otros, Gestión de la educación continua y la capacitación, Manual Moderno, 2004, pp. $11-13$.

[6]Vega García, Rosario: “Aplicación y evaluación de las tecnologías de la información y la comunicación en la educación”, en Evaluación y Tendencias en la Educación Profesional Técnica en Hispanoamérica en el siglo XXI, Colegio Nacional de Educación Profesional Técnica y Agencia Española de Cooperación Internacional, México, D. F., junio 2004. (CD-ROM). 